\title{
Multidisciplinary Integrated Framework for the Optimal Design of a Jet Aircraft Wing
}

\author{
Laura Mainini and Paolo Maggiore \\ Aerospace Engineering Department, Politecnico di Torino, 10129 Torino, Italy \\ Correspondence should be addressed to Paolo Maggiore, paolo.maggiore@polito.it
}

Received 15 November 2011; Revised 8 February 2012; Accepted 8 February 2012

Academic Editor: Kenneth M. Sobel

Copyright ( $) 2012$ L. Mainini and P. Maggiore. This is an open access article distributed under the Creative Commons Attribution License, which permits unrestricted use, distribution, and reproduction in any medium, provided the original work is properly cited.

The preliminary design of a jet aircraft wing, through the use of an integrated multidisciplinary design environment, is presented in this paper. A framework for parametric studies of wing structures has been developed on the basis of a multilevel distributed analysis architecture with a "hybrid strategy" process that is able to perform deterministic optimizations and tradeoff studies simultaneously. The particular feature of the proposed multilevel optimization architecture is that it can use different set of variables, defined expressly for each level, in a multi-level scheme using "low fidelity" and "high fidelity" models, as well as surrogate models. The prototype of the design environment has been developed using both commercial codes and in-house tools and it can be implemented in a geographically distributed and heterogeneous IT context.

\section{Introduction}

Aerospace engineering is characterized by great complexity of the systems to be designed and managed. This complexity is due basically to the fact that large-scale systems are considered as well as design problems characterized by strong interactions among the subsystems and the disciplinary analyses involved. Moreover some design requirements are particularly demanding, especially for aircraft industries: in particular, the need to maintain competitiveness, fundamentally in terms of design quality and of reduction of the time to market, is a critical issue. A good response to these requirements can be found through the use of the tools and methodologies gathered under the name of Concurrent Engineering (CE). These strategies focus on the integration of the design and development phases, being the crucial point that has to be solved in order to accomplish competitiveness requirements. The translation of this strategy into an integrated design language is known as Multidisciplinary Analysis and Optimization (MAO) methodologies. These techniques have been developed to achieve a global optimum for multidisciplinary systems [1$3]$. Designers and researchers have noted that the so-called "all-at-once" technique suffers from practical limitations and is confined to only small, simplified problems, at a conceptual level. Designers have investigated optimization methods in several engineering fields over the last few years. These methods include decomposition strategies, evolutionary and mimetic algorithms, approximation techniques, response surfaces methodology, robust analysis, and reliability-based optimization. Good surveys about these topics can be found in [4-8], while an example of the use of response surfaces for a first generation MDO can be found in [9].

The so called multilevel MDO techniques have also been proposed among the wide set of possible solutions that can be found in the literature. These techniques allow the large, complex systems involved at higher design levels (i.e., the preliminary design) to be globally optimized. This approach has proved to be successful as it mirrors the way a complex system design is actually accomplished by an engineering team. A promising multilevel MDO technique is evaluated and applied in this study in which a global strategy guides the topology parameters at the first level [10]. This technique has been evaluated by applying it to the design optimization of an aircraft wing. A suitable multidisciplinary integrated design environment has been developed in order to manage this application and the work proposed belongs definitely to the so-called third-generation MDO $[5,8]$. This environment is 


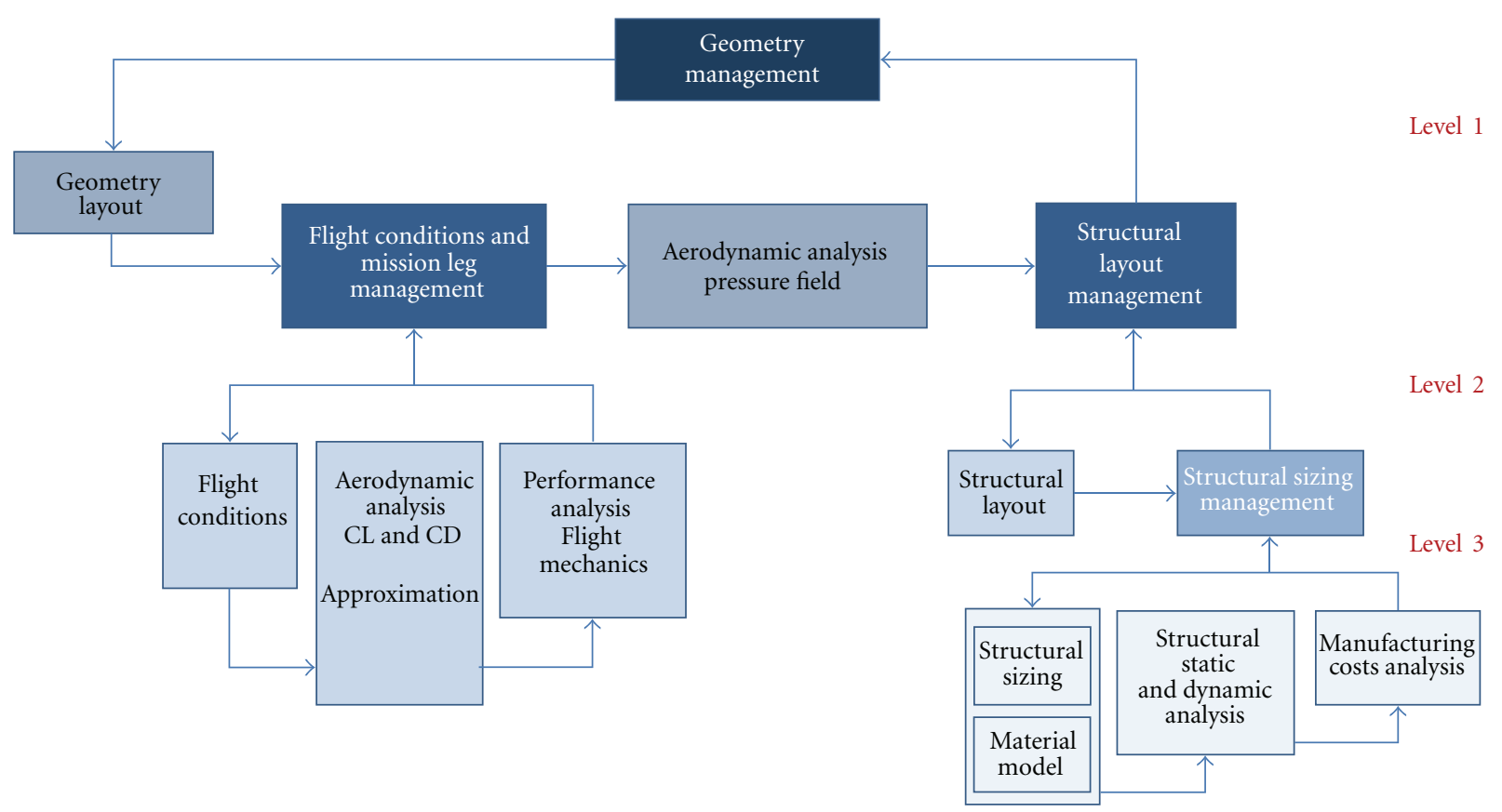

FIGURE 1: Multilevel wing design process.

able to address two crucial key issues of concurrent design: interdisciplinarity and multidisciplinarity.

Multidisciplinarity means the ability to deal with several different disciplines, that concern the design from the initial phases, in the same environment. In order to conduct such a disciplinary integration, a proper wing design framework has been developed and great effort has been dedicated to the implementation of several different parametric disciplinary models. The interdisciplinarity issue instead concerns the ability to manage the large number of variables that are usually shared by the different disciplines involved in the design process flow, while attempting to avoid feedback and improve performance as much as possible. A multilevel distributed analyses architecture is proposed here for this purpose. This architecture manages variables and models and distributes the process over three levels.

A wing design process model is presented as a test application. Section 2 provides the description of what is referred to as the architecture therefore the approach used to accomplish interdisciplinarity requirements is shown. Section 3 presents a detailed investigation of the multidisciplinarity key point, and the set of parametric models with the associated analyses is described. The test and validation results that show the environment works well can be found in Section 4. Finally, Section 5 sums up the main conclusions.

\section{The Architecture}

As previously mentioned, one of the two key issues that have to be addressed goes under the name of interdisciplinarity. The integrated wing design environment here presented proposes an architecture that is suitable for variables models and process management. The design flow has been organized and distributed over a multilevel analysis architecture. First, it is possible to identify an external main loop that manages the geometrical design variables, and aerodynamics and mission analysis variables. It works at a conceptual design level. A bilevel subarchitecture was then conceived to carry out parametric studies of semimonocoque wing structures. According to the concept proposed by Hansen and Horst [10], it is possible to identify an outer loop that deals with the parameters that describe the global structural configuration of the aircraft wing (i.e., the number and position of the structural parts), and an inner loop that manages a group of variables called "sizing parameters," which defines the structures in detail. This architecture can also be referred to as a "hybrid strategy" and it is characterized by the capacity to use different sets of variables for each level.

Thus, as it is possible to see in Figure 1, three levels can be distinguished in the flow management process. The most external loop, also referred to as the level one, deals with a geometry configuration and mission variables. It proposes the geometry layout and initial values for flight conditions for a certain mission programme. A second level can also be found, the so-called level two. Here it is possible to see the inner loop that manages the mission variables which optimize the performances and the first inner loop related to the structural optimization. The latter only manages the structural layout variables and delegates the management of the sizing variables to another, more internal, loop. Finally, such most internal loop, also named level three, performs the structural sizing and optimizes the materials and dimensions of the structural layout proposed by the external structural loop. In short, from the point of view of the objective function level, an overall geometry configuration is defined on the basis of the analysis of the mission requirements. 
The next level allows an optimal configuration of the wing, to be obtained in terms of aerodynamic efficiency and fuel consumption, as well as an optimal configuration of the structural layout (number of spars, ribs, and stiffeners). The final level conducts an optimal sizing of the structural layout, through static and modal analysis, by minimizing the weights and maximizing the overall wing stiffness. Different optimization algorithms can be used for the different tasks. The selection criteria for design exploration techniques and optimization algorithms are obviously related to the design space characteristics of each task. Both traditional gradient based and evolutionary algorithms are used for the optimum search strategies. This architecture is implemented using the SIMULIA I-Sight environment. Because of its capability to host different software components and to enable a substantial pragmatic fast mapping of the variables, this instrument has been identified as appropriate for the purposes of the present investigation.

\section{The Framework}

The second key issue outlined in the introduction goes under the name of multidisciplinarity, which means the capability to host and integrate different disciplinary analysis tools in the same environment. A suitable geometric model framework has been developed in order to address this crucial point. Such a framework operates first on the analysis of the aircraft mission, and the obtained information is then used to drive the subsequent design phases: performance, aerodynamics, and structural analysis.

First, a geometry configuration is proposed to the mission management loop. The variables related to flight conditions (such as altitude, angle of attack, and airspeed) are also considered. The aerodynamic analysis to evaluate the drag coefficient $\left(C_{D}\right)$ and lift coefficient $\left(C_{L}\right)$ is performed using a particular "mixed fidelity" response surface that has been evaluated by combining results from Vortex Lattice Method simulations and Computational Fluid Dynamics (CFD) analysis on the basis of a commercial finite volumes Navier-Stokes solver code, StarCCM+ by CDADAPCO. A brief description of this method is provided in Section 3.3. Once the aerodynamic force coefficients have been evaluated, a performance analysis and optimization are carried out throughout the evaluation of the implemented flight mechanics relations. A Vortex Lattice Method (VLM) analysis is performed, on the basis of the optimized mission, in order to determine the aerodynamic pressure field that has to be remapped onto the structural Finite Elements (FEs) discretization as aerodynamic load. Thus, static and modal structural analyses are involved and a fully parametrical finite element model of the structural elements of the wing is generated and optimized in terms of mass minimization and stiffness maximization, with respect to the number of structural components, their size, materials, positions, and dimensions. However, despite the different disciplinary analyses that are conducted, the core elements of the framework are the parametric models.

Fully parametric models of the wing have been implemented for all the disciplines involved, in order to satisfy

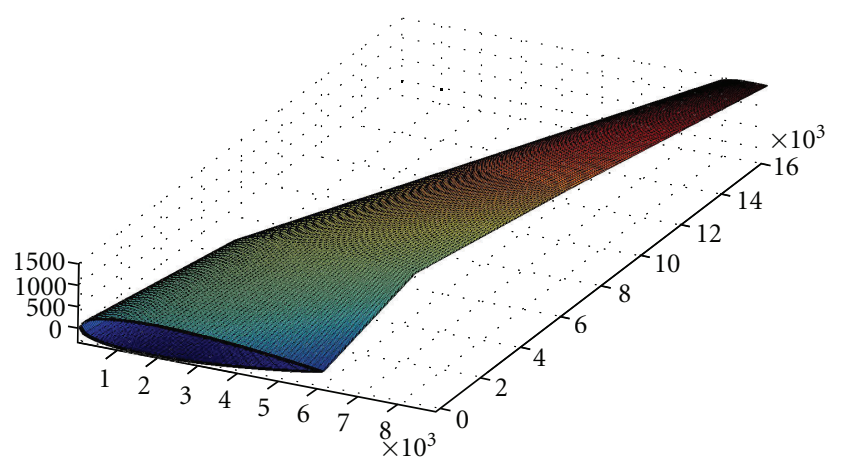

Figure 2: Geometry layout of the wing (sizes in $\mathrm{mm}$ ).

automation requirements of the design process. These models require a great deal of effort to develop the environment. Their importance is surely crucial. In fact, if the parametric models and the disciplinary analysis codes are not carefully developed and properly defined, even the most powerful architecture is completely useless from the MDO point of view. In short, if multidisciplinarity is not guaranteed, the interdisciplinarity issue cannot be addressed, and vice versa.

The several disciplinary wing models that have been examined, which differ from the fidelity and parametrical definition point of view, are described in little more detail hereafter. Special care has been taken in the structural analysis discipline, as these models are the more particularized.

3.1. Geometry of the Layout Model. This model represents the basic reference geometry layout description of the whole design process. The wing geometry is described in terms of generative airfoil parameters, characteristic wing angles (sweep, dihedral, and twist angles), and typical dimensional values, such as aspect ratio, wing span, and taper ratio. A set of 20 geometry variables therefore describes the geometry design space in level one. The wing surface is generated by interpolating of the different generative wing sections, which are parametrically defined on the basis of airfoils camber, maximum thickness, and their percentage chord positions. An indicative representation of a wing geometry described by such a model is shown in Figure 2.

3.2. Geometry of the VLM Aerodynamic Analysis Model. The geometry of the model for the VLM aerodynamic analysis is generated on the basis of the previous one. A 2D model reduction of flat panels, which represent the planform of the wing, has been implemented for the VLM analysis.

3.3. Geometry of the Finite Volumes CFD Analysis Model. Starting from the geometry layout description of the wing, functions that describe the classical NURBS (Non Uniform Rational B-Spline) analytical formulation are used to generate the surfaces that define the external wing geometry. Then, an IGES format file storing the wing body representation, is produced and used as a base to generate the 3-dimensional finite volume grid. The fluid domain is so discretized and 
TABLE 1: Materials database.

\begin{tabular}{|c|c|c|c|c|c|c|c|c|}
\hline \multicolumn{9}{|c|}{ Material DB } \\
\hline \multicolumn{2}{|c|}{ Aluminium alloys } & $\mathrm{E}[\mathrm{MPa}]$ & & $v$ & & & & $\rho\left[\mathrm{kg} / \mathrm{m}^{3}\right]$ \\
\hline 6060 & isot1 & 69000 & & 0,25 & & & & 2,42 \\
\hline 7075 & isot2 & 72000 & & 0,23 & & & & 2,51 \\
\hline 2024 & isot3 & 73000 & & 0,25 & & & & 2,64 \\
\hline \multicolumn{2}{|c|}{ Composites } & E11 [MPa] & $\mathrm{E} 22[\mathrm{MPa}]$ & $v$ & G12 [MPa] & $\mathrm{G} 23[\mathrm{MPa}]$ & $\mathrm{G} 13[\mathrm{MPa}]$ & $\rho\left[\mathrm{kg} / \mathrm{m}^{3}\right]$ \\
\hline Carbon-epoxy & orto 1 & 92600 & 7730 & 0,36 & 3820 & 3820 & 3820 & 1,1 \\
\hline Graphite-ep. 01 & orto 2 & 130000 & 10000 & 0,31 & 4850 & 3620 & 3620 & 1,2 \\
\hline Graphite-ep. 02 & orto3 & 132160 & 8650 & 0,3 & 4120 & 4120 & 4120 & 1,3 \\
\hline
\end{tabular}

the Navier-Stokes equations are solved for the aerodynamic analysis of a real gas, tridimensional turbulent flow.

\subsection{Geometry of the Finite Elements Structural Analysis Model.} The wing is here geometrically described also in terms of structural components. A layout description of the panels, ribs, spars, and stringers is thus provided in terms of number and position, a characteristic dimension is provided, and a structure geometry model is generated for the finite elements discretization. The geometry and dimensions of the spar caps and stringers sections are also considered.

3.5. Material of the Structural Optimization Model. Recent projects have shown an increasing use of composite materials for aircraft structures. By observing the materials that the most recent generation of aircraft are made of, it is possible to see that most of them make use of highly innovative composite materials (Boeing 787 "Dreamliner", Airbus A350, and the well-known Typhoon Eurofighter are recent examples). It is also possible to find several references that testify this trend [11-15]. A fully parametric material description has been implemented to describe the physical and the mechanical properties that are necessary to conduct the structural calculation considering different load conditions and design weight requirements. The model covers both metals and composites (orthotropic materials); in the latter case, an algorithm defines a lay-up method of laminates used for plate and shell parts and pays particular attention to solving the orientation and the laminating sequence problems of orthotropic materials. The material database is shown in Table 1, while Figures 3 and 4 display an example of the property distribution of different materials.

3.6. Finite Element Discretization for Structural Analysis. A fully parametric FEM model of the wing has been implemented. This model allows a detailed control of the structural discretization elements, in terms of number, geometry, dimensions, identification, and properties. Each structural feature (leading-edge, trailing edge, spars, ribs, stringers, pressure, and suction side) is controlled by a flag which allows different configurations and analysis models to be obtained. The consequent full traceability of each element and the associated structural components allow the analyses, which are characterized by different levels of fidelity, to be managed.

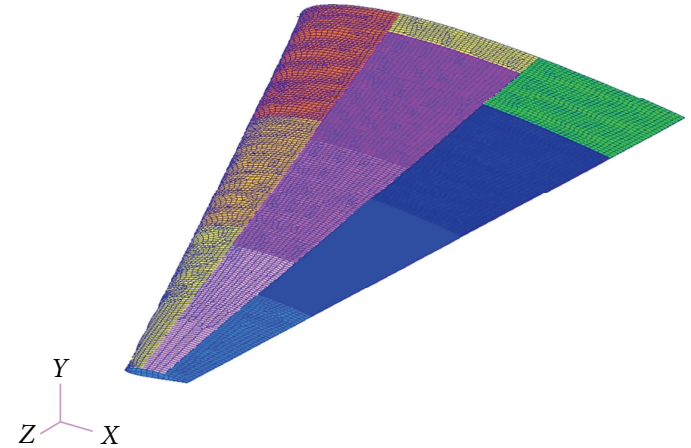

FIGURE 3: Material property distribution for wing panels. There is a different material for a different component of the colour palette. So the several colours simply display the possibility to have a nonuniform material design for a wing, but to tailor different solutions for different structural components.

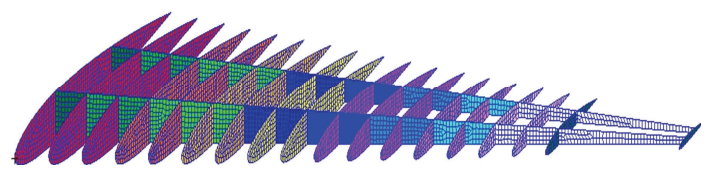

FIGURE 4: Material property distribution for spars and ribs. There is a different material for a different component of the colour palette. So the several colours simply display the possibility to have a nonuniform material design for a wing, but to tailor different solutions for different structural components.

The model has been implemented using MSC-Patran Command Language for the complete parameterization of the structural model. Ribs, spars (Figure 5), and the external wing panels (Figure 6) are modelled using shell elements, while the stiffeners (Figure 7) are discretized with beam elements. In the present paper, only the central wing box has been considered for the structural analysis (Figure 8). The aerodynamic loads obtained from the previous analysis are mapped directly onto the structural model as pressure on the upper and lower wing surfaces. The fuel weight is applied in the appropriate positions as a force per unit of area. Concentrated loads, such as engines and landing gear struts, can be modelled and optimized according to the load positioning. Different loading factors can be also considered 


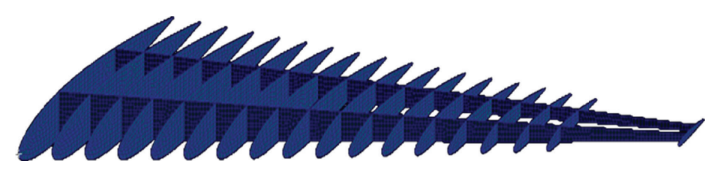

FIGURE 5: FEM panel model with shell elements.

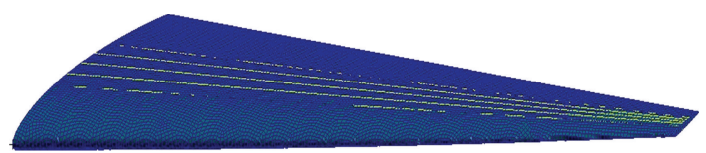

FIGURE 6: FEM ribs and spars model with shell elements.

TABLE 2: Level 1 results summary.

\begin{tabular}{lc}
\hline & Geometry Loop \\
\hline & Airplane data \\
\hline Weight & $686700 \mathrm{~N}$ \\
\hline Geometry variables & Best \\
\hline Dimensions: & $17,5 \mathrm{~m}$ \\
Semispan & $8 \mathrm{~m}$ \\
Root chord & $81,34 \mathrm{~m}^{2}$ \\
Reference surface & 0,16 \\
Taper ratio & 15,06 \\
Aspect ratio & \\
Angles: & $25^{\circ}$ \\
Sweep angle & $5^{\circ}$ \\
Dihedral angle & $3^{\circ}$ \\
Twist angle & \\
Air foil data: & 4 \\
Number of air foil & Position \\
\hline Air foil 4-digit NACA & $0 \%$ span \\
\hline NACA1412 & $30 \%$ span \\
NACA2412 & $60 \%$ span \\
NACA2412 & $100 \%$ span \\
NACA2410 &
\end{tabular}

to model different flight manoeuvre conditions along the three coordinate directions.

3.7. Discretized Model for 2D VLM Aerodynamic Analysis. The VLM model has been defined for aerodynamic analysis in terms of the discretization and parameterization of the number of line vortices and source lattices used to model the fluid behaviour. The calculation is done using the AVL (Athena Vortex Lattice) open source code.

3.8. Discretized Model of the Fluid Domain for 3D CFD Analysis. A parametric model to discretize the fluid domain has been built. A parametric code has been implemented to generate a structured calculus grid and adapt it to the different wing geometry configurations, considering different flight conditions (Figure 9). The implemented process uses the CD-Adapco StarCCM+ code, which is driven by a set of

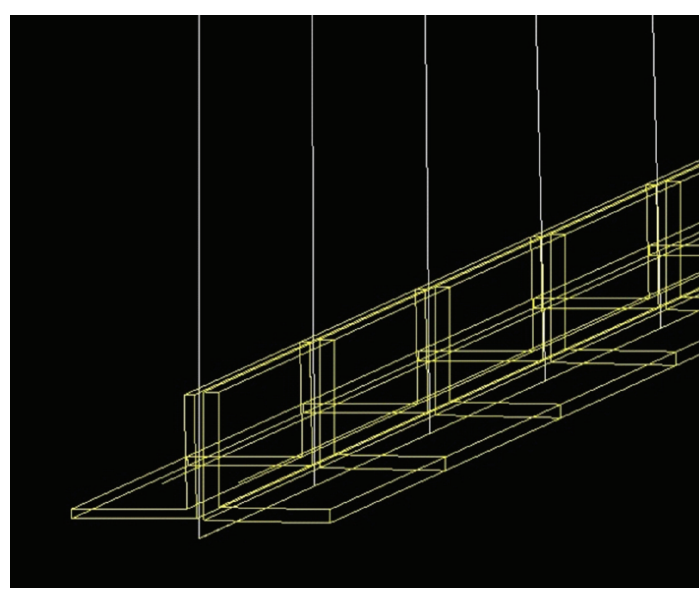

FIGURE 7: FEM stiffeners model with beam elements.
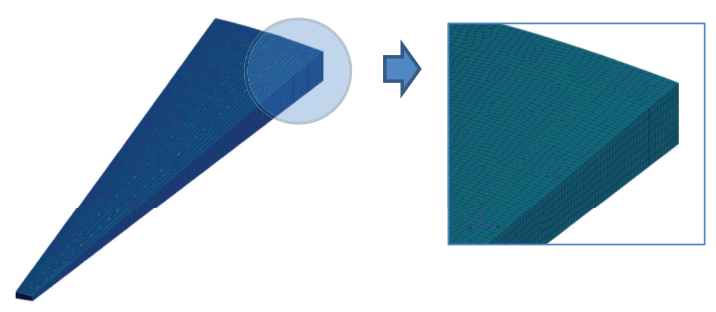

FIGURE 8: FEM model of the wing box.

TABLE 3: Level 2, mission optimization loop results summary.

\begin{tabular}{lcc}
\hline \multicolumn{2}{c}{ Mission optimization } \\
\hline$W_{\text {fuel }}$ & Performance objectives \\
$E$ & Mission condition & Max \\
\hline \multicolumn{2}{c}{} \\
\hline mission phase & cruise \\
Range & $5120 \mathrm{~nm}$ \\
Altitude & $10000 \mathrm{~m}$ \\
$T$ & $223,15 \mathrm{~K}$ \\
$a$ & $299,46 \mathrm{~m} / \mathrm{s}$ \\
$p$ & $2,64 \mathrm{E}+04 \mathrm{~Pa}$ \\
rho & $0,4127 \mathrm{~kg} / \mathrm{m}^{3}$ \\
\hline Mission variables & Best \\
\hline Airspeed & $279,42 \mathrm{~m} / \mathrm{s}$ \\
Alfa & $6,88^{\circ}$ \\
$C_{L}$ & 0,96 \\
$C_{D}$ & 0,04 \\
Re & 30346949,63 \\
$M$ & 0,93 \\
\hline Optimum objective variables & \\
\hline$E$ & 21,97 \\
$W_{\text {fuel }}$ & $140280 \mathrm{~N}$ \\
\hline
\end{tabular}

recorded Java macros. Figure 10 shows an example related to a velocity field at a specific section of the wing. 
TABLE 4: Level 2, structural layout loop results summary.

\begin{tabular}{|c|c|}
\hline \multicolumn{2}{|c|}{ Structural layout optimization } \\
\hline \multicolumn{2}{|c|}{ Structural objectives } \\
\hline Manufacturing cost & $\min$ \\
\hline Structural mass & $\min$ \\
\hline Maximum displacement & $\min$ \\
\hline \multicolumn{2}{|c|}{ Layout data } \\
\hline Number of engines & 1 \\
\hline Engine mass & $2570 \mathrm{~kg}$ \\
\hline Number of landing gears & 0 \\
\hline Landing gear mass & 0 \\
\hline Layout variables & best \\
\hline \multicolumn{2}{|l|}{ Structural elements: } \\
\hline Number of ribs & 18 \\
\hline Number of spars & 2 \\
\hline \multicolumn{2}{|l|}{ Loads position: } \\
\hline Engine position & 4 rib\# \\
\hline \multicolumn{2}{|l|}{ Material data: } \\
\hline materials & composite \\
\hline number of layers & 10 sym \\
\hline
\end{tabular}

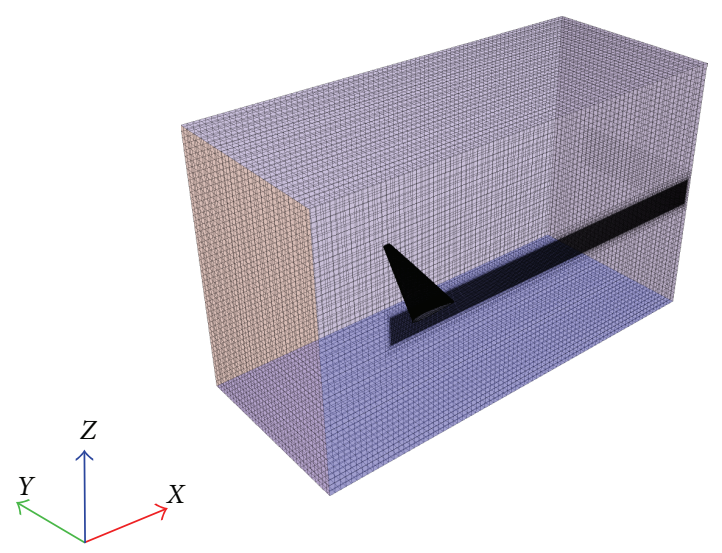

FIGURE 9: Discretized domain for CFD analysis.

3.9. Surrogate Models. The use of surrogate models is currently a fundamental step in reducing computational costs. The metamodel here proposed is a first rough tentative to approach the possibility to use a multifidelity methodology to obtain an approximation for the response of the analysis. The surrogate model has been built as follows: a design space has been defined as determined by the set of geometry and flight conditions variables (a 20D space is explored); the exploration of the design space has been performed according to a DOE (Design of Experiments) process based on an orthogonal array algorithm; values of $C_{D}$ and $C_{L}$ for each design point have been evaluated mixing the low fidelity and high fidelity analysis so that the $90 \%$ of the sample points has been calculated using the VLM model, while the $10 \%$ by the evaluation of the StarCCM+ model. The values so obtained were used to build a quadratic response surface that represents the actual approximation tool to determine

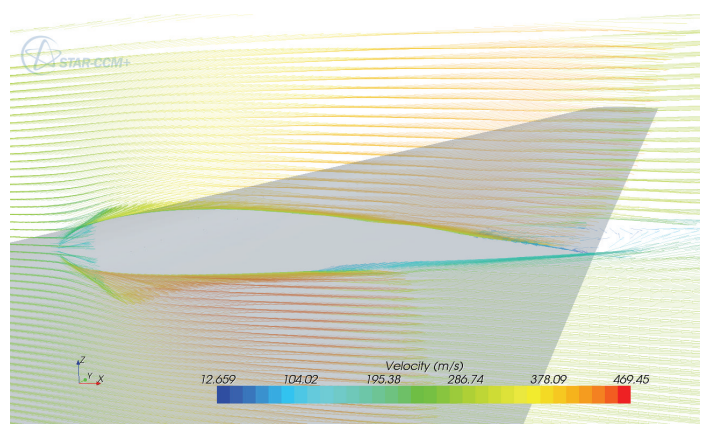

FIGURE 10: Velocity field along a section station of the wing.

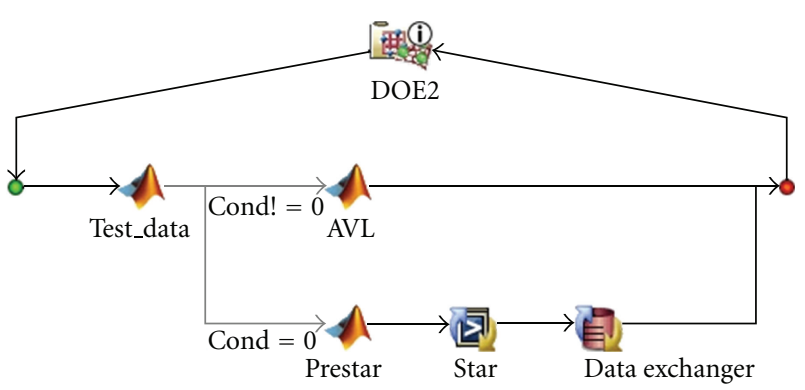

FIGURE 11: Surrogate model generating process involving different fidelity models. AVL represents the low-fidelity model and is used to evaluate the $90 \%$ of the sampled points of the design space. StarCCM+ is used for the high-fidelity model and the $10 \%$ of the points is so evaluated.

$C_{D}$ and $C_{L}$ for the performance evaluation task. The scope consists in obtaining a certain implicit correction of the low fidelity results by the involvement of a small portion of high fidelity evaluations while creating the response surface. It has represented a rough tentative done in this first phase just to have a tool to reduce computational cost. A particular process architecture was implemented in order to obtain an approximated aerodynamic model (Figure 11). This approximated aerodynamic model is created using the RS algorithm implemented in the I-Sight approximation tool, as shown in Figure 11. More sophisticated surrogate model methodologies will be studied in the next future. The obtained surrogate model has been used in the first two levels of the implemented MDO process.

The wing design process has been implemented using SIMULIA I-Sight software. A collection of Matlab functions allows mission analysis to be performed, and certain design phases (e.g., pressure distribution mapping from aerodynamic model to structural model) to be interfaced in a suitable manner.

\section{Testing and Validation}

The integrated design environment here proposed has been tested by simulating the wing design process for a civil midrange aircraft. Tables $2-5$ show the results. The variables and values are summarized according to the distribution over the three levels of the proposed architecture. 
TABLE 5: Level 3, structural sizing loop results summary.

(a) Structural Sizing Optimization

\begin{tabular}{|c|c|}
\hline \multicolumn{2}{|c|}{ Sizing data } \\
\hline Stringer section & L section \\
\hline Spar cap material & Aluminium \\
\hline Stringer material & Aluminium \\
\hline Sizing variables & Best \\
\hline \multicolumn{2}{|c|}{ Spar caps (Figure 12 shows the legend for element position) } \\
\hline h13 & $21,24 \mathrm{~mm}$ \\
\hline $\mathrm{h} 24$ & $22,29 \mathrm{~mm}$ \\
\hline Base13 & $22,19 \mathrm{~mm}$ \\
\hline Base24 & $22,08 \mathrm{~mm}$ \\
\hline Thickness h13 & $1,72 \mathrm{~mm}$ \\
\hline Thickness h24 & $2,34 \mathrm{~mm}$ \\
\hline Thickness base13 & $1,62 \mathrm{~mm}$ \\
\hline Thickness base 24 & $2,64 \mathrm{~mm}$ \\
\hline Stringers & L section \\
\hline h top & $22,02 \mathrm{~mm}$ \\
\hline h bottom & $15,86 \mathrm{~mm}$ \\
\hline Base top & $21,41 \mathrm{~mm}$ \\
\hline Base bottom & $20,30 \mathrm{~mm}$ \\
\hline Thickness h top & $2,98 \mathrm{~mm}$ \\
\hline Thickness h bottom & $2,57 \mathrm{~mm}$ \\
\hline Thickness base top & $1,86 \mathrm{~mm}$ \\
\hline Thickness base bottom & $2,66 \mathrm{~mm}$ \\
\hline Number of stringers & 3 \\
\hline \multicolumn{2}{|l|}{ Spars position } \\
\hline 1 & $25,98 \%$ chord \\
\hline 2 & $62,11 \%$ chord \\
\hline \multicolumn{2}{|l|}{ Ribs position } \\
\hline 1 & $0 \%$ span \\
\hline 2 & $5 \%$ span \\
\hline 3 & $10 \%$ span \\
\hline 4 & $15 \%$ span \\
\hline 5 & $20 \%$ span \\
\hline 6 & $25 \%$ span \\
\hline 7 & $30 \%$ span \\
\hline 8 & $35 \%$ span \\
\hline 9 & $40 \%$ span \\
\hline 10 & $45 \%$ span \\
\hline 11 & $50 \%$ span \\
\hline 12 & $55 \%$ span \\
\hline 13 & $60 \%$ span \\
\hline 14 & $65 \%$ span \\
\hline 15 & $70 \%$ span \\
\hline 16 & $75 \%$ span \\
\hline 17 & $80 \%$ span \\
\hline 18 & $100 \%$ span \\
\hline
\end{tabular}


(b) Composites stacking sequence characteristics

\begin{tabular}{|c|c|c|c|c|c|c|c|c|c|c|}
\hline Layers thickness & & & & & & & & & & \\
\hline Leading edge & $0,3 \mathrm{~mm}$ & & & & & & & & & \\
\hline Top surface & $0,3 \mathrm{~mm}$ & & & & & & & & & \\
\hline Bottom surface & $0,3 \mathrm{~mm}$ & & & & & & & & & \\
\hline Trailing edge & $0,3 \mathrm{~mm}$ & & & & & & & & & \\
\hline Spars & $0,3 \mathrm{~mm}$ & & & & & & & & & \\
\hline Ribs & $0,3 \mathrm{~mm}$ & & & & & & & & & \\
\hline Layers orientation $\left[{ }^{\circ}\right]$ & 1 & 2 & 3 & 4 & 5 & 6 & 7 & 8 & 9 & 10 \\
\hline Leading edge & 45 & 45 & 90 & 45 & 90 & 90 & 45 & 90 & 45 & 45 \\
\hline Top surface & 90 & 45 & 45 & 90 & 45 & 0 & 90 & 90 & 0 & 45 \\
\hline Bottom surface & 90 & 45 & 90 & 90 & 0 & 45 & 0 & 90 & 45 & 90 \\
\hline Trailing edge & 90 & 0 & 45 & 0 & 45 & 45 & 45 & 45 & 90 & 0 \\
\hline Spars & 45 & 45 & 90 & 90 & 90 & 45 & 45 & 45 & 90 & 0 \\
\hline Ribs & 45 & 45 & 90 & 45 & 45 & 0 & 0 & 0 & 0 & 0 \\
\hline
\end{tabular}

(c) Optimum objective variables

\begin{tabular}{lc}
\hline Manufacturing cost & $4,35 \mathrm{E}+07$ \\
Structural mass & $2077 \mathrm{~kg}$ \\
Maximum displacement & $1603 \mathrm{~mm}$ \\
\hline
\end{tabular}

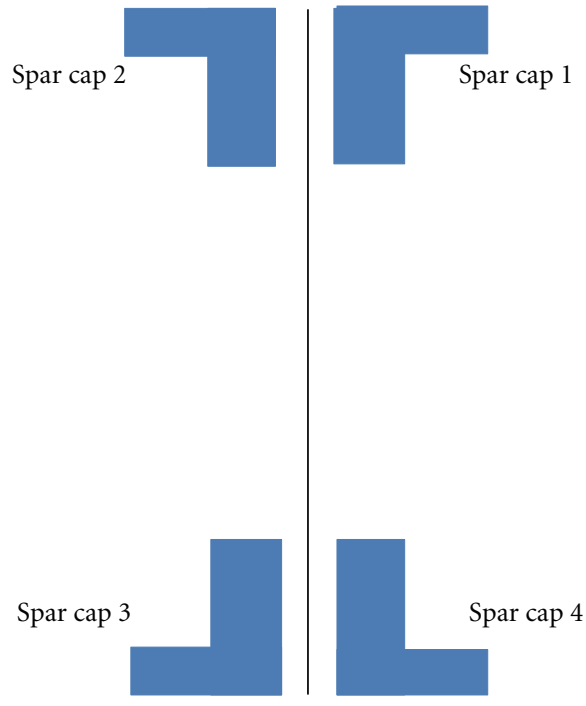

FIgURE 12: Legend for spar caps position.

Table 2, which is related to level one (the most internal loop), shows the behaviour of the geometry variables. Here, it is possible to find their values at the optimum design point. As shown, four generative airfoils are used to determine the spanwise wing section behaviour. A NACA 4-digit type airfoil has been chosen and parameterized according to the classical NACA 4-digit code definition. The span-wise position of the airfoils is also optimized.

The data related to the process managed at level two can be found in Tables 3 and 4 . The mission phase (Table 3 ) here tested is cruising and it is defined on the basis of the environmental conditions, altitude, and the range to be covered. Airspeed and angle of attack are optimized for a certain defined situation in order to maximize aerodynamic efficiency and minimize fuel consumption, which are easily translated into the amount and weight of fuel that has to be stored onboard. The structural layout management loop (Table 4) defines a certain load condition on the basis of the concentrated load data, aerodynamic pressure distribution in the optimized flight condition, and the fuel load distribution along wing tanks. The layout variables include the load position and the number of structural components with their material assignment.

Table 5, related to level three (also referred to as the structural sizing loop), shows the value assumed by the structural sizing variables at the optimum point of the design space. Such an optimum point maximizes stiffness, minimizing the overall structural mass and the displacement of the wingtip.

As it is possible to see from the values and data in the tables, the environment shows very good behaviour.

\section{Conclusions}

In this paper an integrated design environment for wing design is presented according to multidisciplinary design optimization paradigms. The management of the complexity issue has been addressed through the accomplishment of the multidisciplinarity and interdisciplinarity drivers. According to those key points, great efforts have been dedicated to the implementation of several parametric models for the different disciplinary analyses involved and a multilevel architecture has been proposed and tested for the analysis management purposes. Such multilevel approach allows a good integration the different phases of the wing design and a proper dealing of the complex dataflow among the involved disciplines.

Reduced or surrogate models are needed to reduce computational efforts for aerodynamic performance 
optimization purposes. The mixed approach here presented seems to be promising, even though the algorithm used is quite rough: in fact the management of the variable fidelity analysis is not done in an organic way in the sense that the correction is left as an implicit and not controlled phase. Further studies will be performed on surrogate models using more sophisticated mathematical methods and correction strategies.

The environment has been validated through the simulation of the design process of a wing for a civil midrange aircraft, optimizing the cruise phase of a typical civil transportation mission.

\section{References}

[1] J. J. Alonso, P. LeGresley, and V. Pereyra, "Aircraft design optimization," Mathematics and Computers in Simulation, vol. 79, no. 6, pp. 1948-1958, 2009.

[2] P. Chwalowski, J. A. Samareh, L. G. Horta, D. J. Piatak, and A. R. McGowan, "Efficient multidisciphnary analysis approach for conceptual design of aircraft with large shape change," Tech. Rep. RTO-A T-168, 2009.

[3] W. H. Jones, "Project integration architecture: application architecture," Technical Memorandum NASA/TM-2005-213611, National Aeronautics and Space Administration, Glenn Research Center, Cleveland, Ohio, USA, 2005.

[4] S. Shan and G. G. Wang, "Survey of modelling and optimization strategies for high-dimensional design problems," in Proceedings of the 12th AIAA/ISSMO Multidisciplinary Analysis and Optimization Conference, British Columbia, Canada, September 2008.

[5] J. Sobieszczanski-Sobieski and R.T. Haftka, "Multidisciplinary aerospace optimization: survey of recent developments," Tech. Rep. AIAA 96-0711, Structural Optimization, 1997.

[6] J. Agte, O. de Weck, J. Sobieszczanski-Sobieski, P. Arendsen, A. Morris, and M. Spieck, "MDO: assessment and direction for advancement-an opinion of one international group," Structural and Multidisciplinary Optimization, vol. 40, no. 16, pp. 17-33, 2010.

[7] T. W. Simpson, V. Toropov, V. Balabanov, and F. A. C. Viana, "Design and analysis of computer experiments in multidisciplinary design optimization: a review of how far we have come-or not," in Proceedings of the 12th AIAA/ISSMO Multidisciplinary Analysis and Optimization Conference, British Columbia, Canada, September 2008.

[8] X. Chen, L. Yn, W. Luo, L. Xu, Y. Zhao, and Z. Wang, "Research on theory and application of multidisciplinary design optimization of flight vehicles," in Proceedings of the 47th AIAA/ASME/ASCE/AHS/ASC Structures, Structural Dynamics, and Materials Conference, Newport, RI, USA, May 2006.

[9] Y. Kim, Y. H. Jeon, and D. H. Lee, "Multi-objective and multidisciplinary design optimization of supersonic fighter wing," Journal of Aircraft, vol. 43, no. 3, pp. 817-824, 2006.

[10] L. U. Hansen and P. Horst, "Multilevel optimization in aircraft structural design evaluation," Computers and Structures, vol. 86, no. 1-2, pp. 104-118, 2008.

[11] D. C. Walden, "Applications of composites in commercial airplanes. In: structural composites: design and processing technologies," in Proceedings of the 6th Annual ASM/ESD Advanced Composites Conference, ASM International, Detroit, Mich, USA, October 1990.
[12] B. Harmon and S. A. Arnold, "Cost implications of composite materials in military airframes," in Proceedings of the 7th Annual ASM/ESD Advanced Composites Conference, Detroit, Mich, USA, September 1991.

[13] M. C. Niu, Composite Airframe Structures, Comnilit Press, Hong Kong, 1992.

[14] D. H. Middleton, Composite Materials in Aircraft Structures, Longman Scientific and Technical, Essex, UK, 1990.

[15] G. F. Turner, "Advanced composite materials in European aircraft present and future," in Proceedings of the 40th International SAMPE Symposium, pp. 366-380, SAMPE, Covina, Calif, USA, May 1995. 

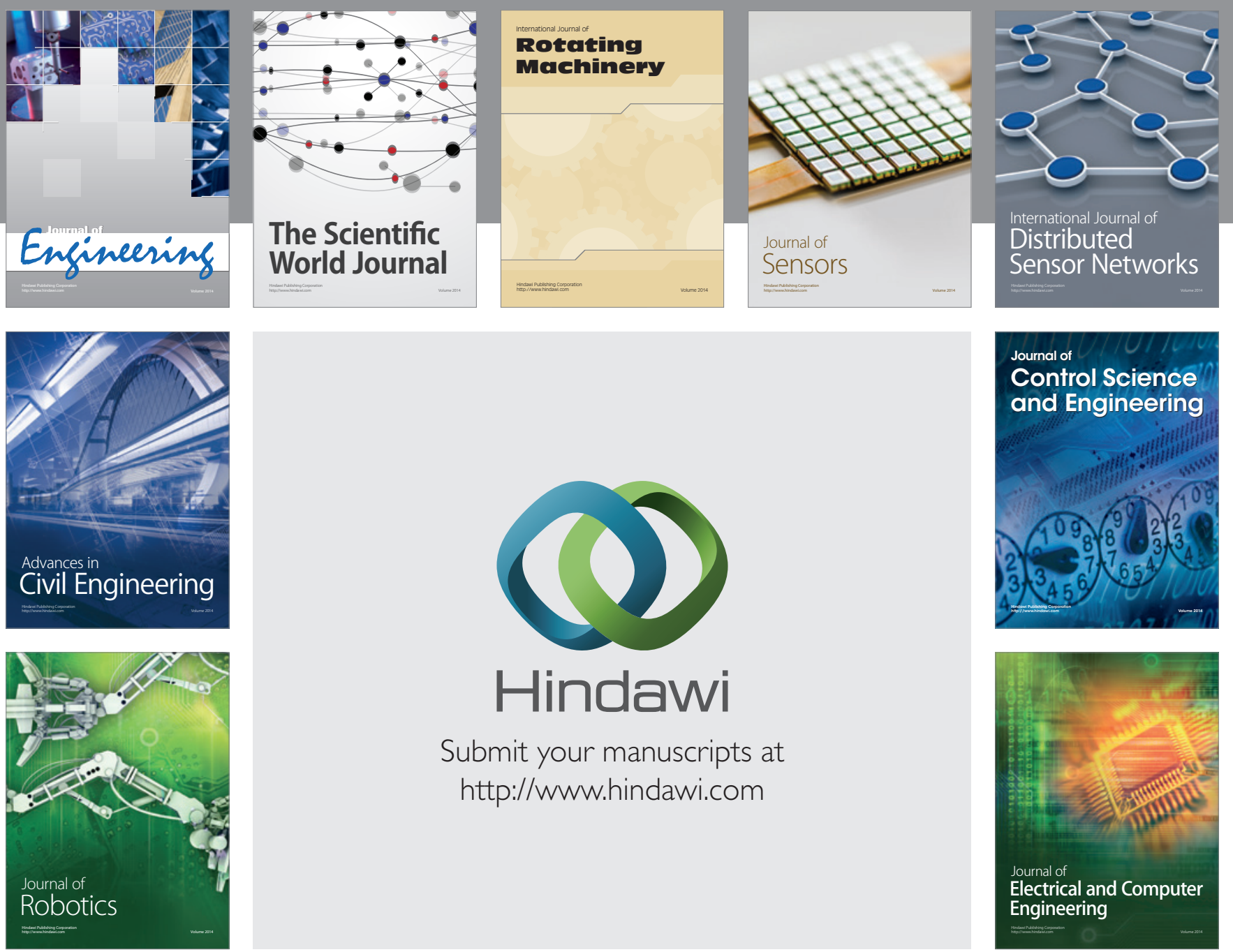

Submit your manuscripts at

http://www.hindawi.com
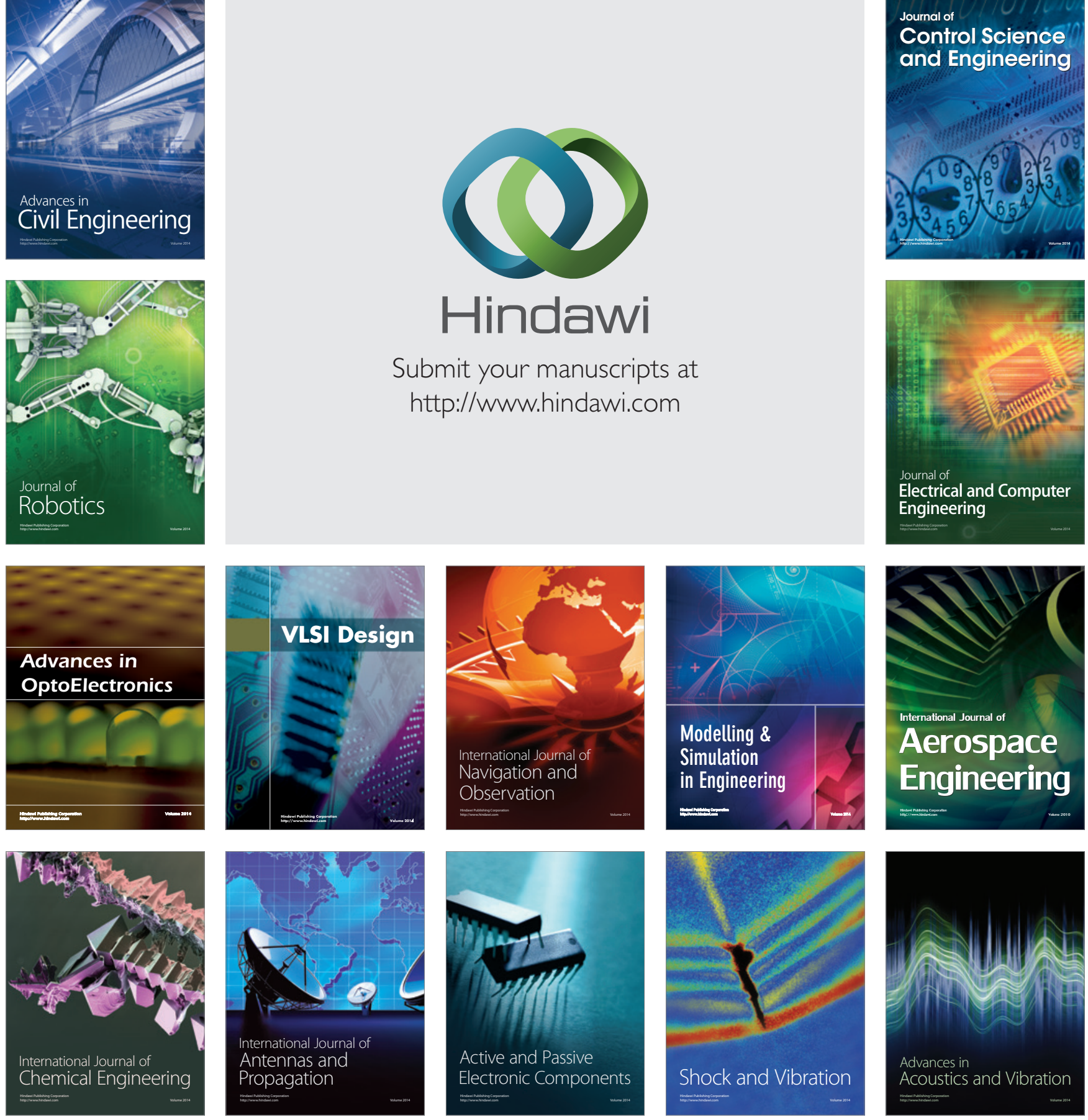